\title{
Hidatidosis musculoesquelética: una localización atípica
}

\author{
M. PLEGUEZUELO NAVARRO, E. M. IGLESIAS FLORES, J. L. DOMÍNGUEZ \\ JIMÉNEZ, A. GONZÁLEZ GALILEA, J. A. ABAD LARA', C. JIMÉNEZ SÁNCHEZ', \\ J. F. DE DIOS VEGA \\ Servicios de Aparato Digestivo, 'Traumatología y Cirugía Ortopédica. Hospital \\ Universitario Reina Sofía. Córdoba
}

\author{
MUSCULOSKELETAL HYDATIDOSIS: AN UNUSUAL LOCATION
}

\begin{abstract}
RESUMEN
La hidatidosis es una zoonosis con una elevada prevalencia aún en nuestro medio. Generalmente afecta a hígado y pulmón, debiendo considerarse la afectación músculo-esquelética una localización atípica de la enfermedad. Presentamos el caso de un paciente con lesiones en cresta iliaca y glúteo medio izquierdos, con fistulización espontánea a través de la piel. Se realizó tratamiento combinado con un ciclo de albendazol previo a la intervención y exéresis quirúrgica de la lesión. En el postoperatorio se completaron dos ciclos más de tratamiento con albendazol en un intento de disminuir el riesgo de recurrencia de la enfermedad. El paciente se encuentra asintomático y no ha presentado ninguna complicación hasta la fecha.
\end{abstract}

PALABRAS CLAVE: Quiste hidatídico. Echinococcus. Sistema musculoesquelético. Localización atípica.

\begin{abstract}
Hydatidosis is a zoonosis with a continuing high prevalence in our environment. The most commonly affected organs are the lungs and the liver, with the musculoskeletal location being considered an unusual one. We comment the case of a patient who presented a series of lesions in his left iliac crest and middle left buttock with spontaneous fistulization to the skin surface. In this case a combined treatment was given; prior to the surgical operation we administered a cycle of albendazol. Following removal of the lesion, the patient was given two further cycles of albendazol in order to minimize the risk of a recurrence of the illness. This patient is currently free of any symptoms relating to this illness.
\end{abstract}

KEY WORDS: Hydatic cyst. Echinococcus. Musculoskeletal system. Unusual localization.

Pleguezuelo Navarro M, Iglesias Flores EM, Domínguez, Jiménez JL, González Galilea A, Abad Lara JA, Jiménez Sánchez C, de Dios Vega JF. Hidatidosis musculoesquelética: una localización atípica. An Med Interna (Madrid) 2006; 23: 229-231.

\section{INTRODUCCIÓN}

La hidatidosis es una enfermedad parasitaria causada por el Echinococcus granulosus. En nuestro medio el ciclo biológico se mantiene habitualmente entre cánidos (fundamentalmente el perro) y ungulados domésticos (el más frecuente la oveja), con la posible intervención accidental del hombre al ingerir los huevos de E. granulosus con el agua o los alimentos contaminados o más frecuentemente, por el contacto directo con perros infestados (1). España se encuentra entre los países de Europa con mayor incidencia de la enfermedad en humanos, con tasas variables pero especialmente relevantes en zonas rurales dedicadas a la crianza de ganado ovino. Aunque los órganos que con mayor frecuencia se afectan son el hígado y los pulmones, si el parásito logra atravesar estos filtros los quistes pueden asentar en muy diversas localizaciones. La afectación musculoesquelética por E. granulosus debe ser considerada como una localización atípica y muy poco fre- cuente de esta enfermedad, suponiendo el 1-4\% de los casos de hidatidosis (2). Comunicamos el caso de un varón de 63 años con hidatidosis musculoesquelética, las distintas exploraciones realizadas y el resultado del tratamiento médico-quirúrgico al que fue sometido.

\section{CASO APORTADO}

Varón de 63 años remitido a la consulta de gastroenterología por dolor en vacío y fosa iliaca izquierdos, de características continuas, sin relacionarse con la defecación ni con la ingesta de alimentos. Apreciaba aumento del dolor con la deambulación y la flexión del tronco. Como antecedente médico-quirúrgico destacaba una intervención hacía aproximadamente 10 años de quiste hidatídico en pelvis menor, con afectación de músculo iliaco, psoas y raíz de miembro inferior izquierdo. Entonces se realizó quistoperiquistectomía y tratamiento local con suero hipertónico. Seis años más tarde precisó drenaje y marsupialización de un nuevo quiste en músculo glúteo

Trabajo aceptado: 16 de noviembre de 2005 
izquierdo. Siguió tratamiento farmacológico coadyuvante con albendazol, que se asoció a toxicidad hepática leve, con discreto aumento de los niveles de transaminasas autolimitado tras la suspensión del fármaco, así como alopecia. Otros antecedentes médicos eran cardiopatía isquémica con infarto agudo de miocardio hacía dos años, brucelosis y fractura de clavícula derecha y pelvis hacía 15 años por politraumatismo.

El paciente presentaba buen estado general, estaba afebril y con tensión arterial, frecuencia cardiaca y respiratoria dentro de los límites normales. La auscultación cardiorrespiratoria era normal y la exploración abdominal despertaba dolor moderado a la palpación en vacío y fosa iliaca izquierdos, no apreciándose masas ni visceromegalias. En la exploración de la articulación coxofemoral izquierda no se evidenció alteración alguna, siendo la flexión/extensión, abducción/addución y rotación normales. Maniobras clásicas como Drahmann o Anvil no se mostraron patológicas. El signo de Trendelenburg-Duchene y la marcha fueron normales. La palpación profunda sobre la fosa iliaca despertaba en el paciente clínica álgida. No había alteraciones significativas en los valores de la hematimetría, estudio de coagulación ni bioquímica general, incluyendo niveles de transaminasas, fosfatasa alcalina y gammaglutamiltranspeptidasa. Por los antecedentes y ante la sospecha clínica de enfermedad hidatídica se solicitó la serología de hidatidosis, resultando positiva a título de 1/1280. La radiografía simple de tórax era normal y en la radiografía de pelvis (Fig. 1) se evidenció un patrón de lesión caracterizado por una afectación moteada que englobaba cresta y ala iliaca izquierdas, con focos osteolíticos y sin esclerosis. La afectación ósea se extendía desde espina iliaca anterosuperior hasta tuberosidad iliaca izquierda. La ecografía abdominal mostró en el segmento VI de lóbulo hepático derecho una lesión redondeada calcificada de $2 \mathrm{~cm}$ de diámetro, sin otras alteraciones significativas.

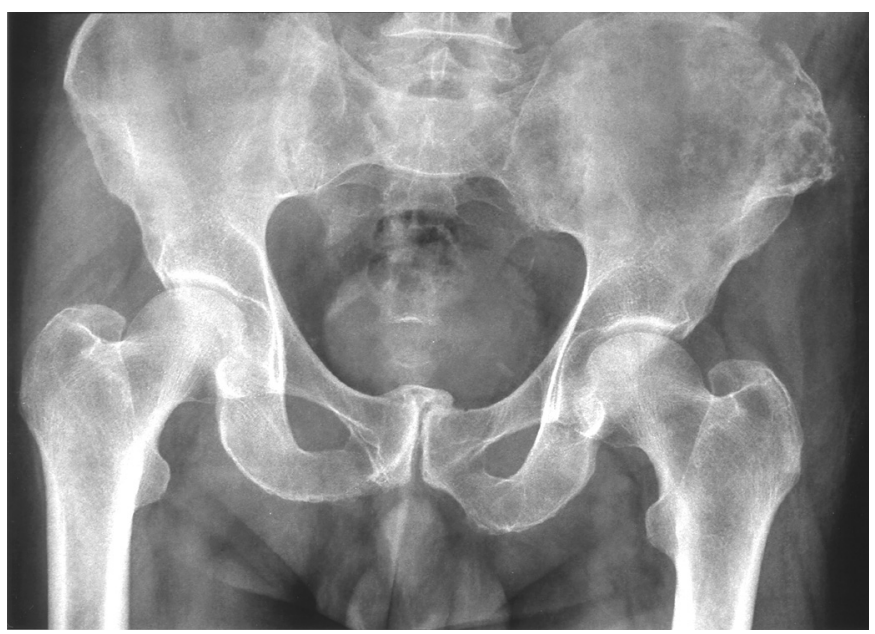

Fig. 1. Rx pelvis.

Los hallazgos de la RMN se muestran en la figura 2. Posterior al músculo cuadrado lumbar izquierdo se apreciaba una imagen quística de 2,5 cm de diámetro máximo; otra lesión multiquística e insuflante en pala iliaca izquierda de 6,4 x 9,2 cm que se extendía inferiormente hasta contactar con borde superior del acetábulo; una tercera se localizaba en el glúteo medio izquierdo, con unas dimensiones de $5 \times 3 \mathrm{~cm}$. Todas las lesiones eran muy sugestivas de afectación musculoesquelética por hidatidosis. Valorado el caso por el Servicio de Traumatología y Cirugía Ortopédica se decide la exéresis del quiste hidatídico localizado en la fosa iliaca izquierda mediante resección de un tercio del íleon izquierdo, así como resección mediante quistoperiquistectomía de las lesiones satélites. Durante la intervención se constató la afectación del músculo psoasiliaco y sartorio izquierdos a nivel de su inserción en ilion, así como de la mus-

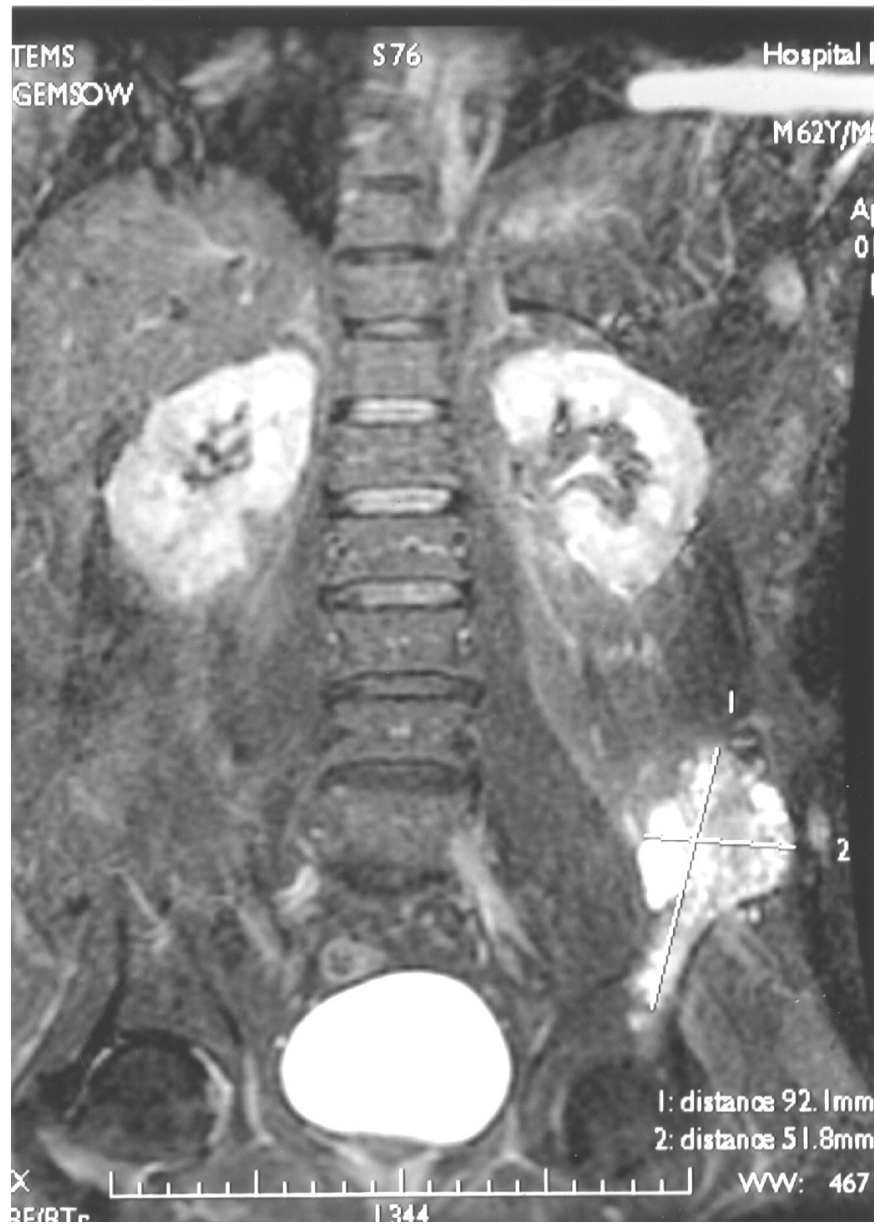

Fig. 2. $R M N$.

culatura glútea izquierda. Antes de la intervención el paciente presentó fistulización espontánea del quiste localizado en la fosa iliaca con drenaje a través de la piel. El estudio histológico de la pieza quirúrgica fue informado como reacción histiocítica pseudoquística a membrana anhista (hidatidosis ósea). Previo a la cirugía y como tratamiento coadyuvante se inició un ciclo con albendazol a dosis de 15 $\mathrm{mgr} / \mathrm{kg} /$ día que se prolongó durante 4 semanas. Estos ciclos se repitieron en dos ocasiones más, con periodos de descanso de dos semanas y monitorización clínica y analítica, sin que aparecieran signos de toxicidad. Durante el postoperatorio presentó fiebre y supuración purulenta de la herida quirúrgica, aislándose en el exudado Proteus mirabilis sensible a Ceftazidima. En las revisiones posteriores el paciente presenta una evolución favorable, presentando sólo cambios postquirúrgicos en la RMN de control.

\section{DISCUSIÓN}

La afectación musculoesquelética por E. granulosus es inusual. Los huesos mas comúnmente afectados son las vértebras, las epífisis de los huesos largos y la pelvis (3). A diferencia de otras localizaciones, en la hidatidosis ósea la parasitosis no adopta forma quística, sino que provoca una infiltración multivesicular sin delimitación clara y sin enquistamiento (4). Los pacientes en estos casos suelen mantenerse asintomáticos durante un largo periodo de tiempo, apareciendo signos y síntomas clínicos de la enfermedad cuando aparece alguna complicación (5). En nuestro caso, la ecografía abdominal mostraba una lesión quística hepática calcificada 
compatible con un quiste hidatídico inviable. Otros autores comunican que la afectación musculoesquelética por hidatidosis generalmente resulta en un hecho aislado, sin afectarse otras vísceras como los pulmones o el hígado (2). La anamnesis es de gran relevancia, y el hecho de vivir en una zona rural y dedicarse a una actividad de riesgo debe reforzar la sospecha de la enfermedad. El diagnóstico se realiza mediante pruebas de imagen como la ecografía y, especialmente, la TC y RMN (6). Ante la sospecha de hidatidosis debe obtenerse confirmación serológica. En la actualidad se realiza mediante una combinación de determinaciones en suero; los más usados son la hemaglutinación indirecta (IHA), la detección de anticuerpos indirectos fluorescentes (IFA) y los inmunoensayos enzimáticos. La sensibilidad global de estas pruebas en pacientes con enfermedad quística varía entre el 64-87\% (7). La detección de anticuerpos en estos casos depende de la localización de las lesiones, la integridad y la vitalidad del quiste. En la hidatidosis ósea suele existir una producción de anticuerpos detectables en suero. La fisurización o ruptura de los quistes provocan una estimulación brusca de anticuerpos, mientras que la calcificación o la muerte de estos se asocian generalmente a seronegatividad. Sin embargo es preciso resaltar que la serología sólo es de utilidad cuando es positi- va, ya que un resultado negativo no excluye el diagnóstico (7). Cuando la afectación es distinta a la hepática y la pulmonar se recomienda la aplicación de técnicas especiales como ELISA, Western Blot o incluso PCR (8). Cuando se detecta una afectación ósea debe descartarse afectación visceral acompañante, al menos mediante una radiografía de tórax y una ecografía abdominal. Ante los hallazgos de las técnicas de imagen deben contemplarse como diagnósticos diferenciales fundamentales el hiperparatiroidismo (que generalmente se presenta con lesiones quísticas múltiples) y el tumor de células gigantes (4). Aunque la exéresis quirúrgica con amplios márgenes se considera como la mejor opción terapéutica, la mayoría de los grupos asocia tratamiento coadyuvante con derivados benzimidazólicos en ciclos pre y postquirúrgicos con intención de mejorar los resultados y disminuir la tasa de recurrencia de la enfermedad $(9,10)$. La cirugía radical provoca una disminución del título de anticuerpos si ésta ha sido eficaz, llegando incluso en ocasiones a hacerse indetectables en el suero. De igual forma, la recurrencia de la enfermedad se seguiría de una elevación de éstos (8). Por ello se recomienda realizar controles serológicos periódicos de los pacientes que tras la intervención quirúrgica son tratados con derivados benzimidazólicos.

\section{Bibliografía}

1. Portús Vinyeta M. Infecciones causadas por cestodos. En: Farreras Valentí P, Rozman C, ed. Medicina Interna. Madrid, Ed. Harcourt 2000, vol II; p. 2770-2776.

2. Merkle EM, Schulte M, Vogel J, Tomczak R, Rieber A, Kern P, et al. Musculoskeletal involvement in cystic echinococcosis: Report of eight cases and review of the literature. Am J Roentgenol 1997; 168: 15311534.

3. Beggs I. The radiology of hydatid disease. AJR 1985; 145: 639-648.

4. Jlidi J, Yaakoubi mt, Ladeb MF, Ben Ayeche ML, Ghannouchi G, Moula T. L'hydatidose osseuse. Ann Pathol 1992; 12: 98-102.

5. Madiwale C, Shenoy A, Joshi A, Hemmadi SS, Bhosale PB. Hydatid cyst of the tibia. J Postgrad Med. 1992; 38: 194-195, 197.

6. Dahniya MH, Hanna RM, Ashebu S, Muhtaseb SA, El-Beltagi A, Badr

$\mathrm{S}$, et al. The imaging appearances of hydatid disease at some unusual sites. Br J Radiol 2001; 74: 283-289.

7. Orhan Z, Kara H, Tuzuner T, Sencan I, Alper M. Primary subcuatenous cyst hydatic disease in proximal thigh: an unusual localization: a case report. BMC Musculoskelet Disord 2003; 4: 25.

8. Biava MF, Dao A, Fostier B. Laboratory diagnosis of cystic hydatic disease. World J Surg 2001; 25: 10-14.

9. García-Álvarez A, Torcal J, Salinas JC, Navarro A, García-Alvarez I, Navarro-Zorraquino M, Sousa R, Tejero E, Lozano R. Musculoskeletal hydatid disease. A report of 13 cases. Acta Orthop Scand 2002; 73: 227 231.

10. Soleto SE. Relapsing infection with hepatic hydatidosis: Problems of surgical treatment. Arch Surg 1991; 30: 609. 